\title{
The New Approach to the Synthesis of Single-Channel Consistent Estimates of the Time Signal Parameters
}

\author{
Oleg V. CHERNOYAROV ${ }^{1,2}$, Yury A. KUTOYANTS ${ }^{1,3}$ and Boris I. SHAKHTARIN ${ }^{4}$ \\ ${ }^{1}$ National Research University "MPEl”, 14, Krasnokazarmennaya st., 111250 Moscow, Russia \\ ${ }^{2}$ International Laboratory of Statistics of Stochastic Processes and Quantitative Finance of National Research Tomsk State University, 36 , \\ Lenin avenue, 634050 Tomsk, Russia \\ ${ }^{3}$ Universite du Maine, Avenue Olivier Messiaen, 72085 Le Mans, France \\ ${ }^{4}$ Bauman Moscow State University 5, Baumanskaya 2-ya st., 105005 Moscow, Russia
}

\begin{abstract}
Based on the introduced modification of a maximum likelihood method we suggest the new technique for synthesis of single-channel quasi-optimal processing algorithms of signals with multiple unknown parameters. We illustrate the proposed approach by the estimation of the appearance time and duration of a rectangular video pulse. We show that unlike technically complex multichannel maximum likelihood estimates, the single-channel quasi-optimal estimates of time signal parameters are consistent for the implementation. We also find the analytical expressions for characteristics of passed estimates which allow us to assess the reasonableness of the application of this or that processing algorithm in each specific case.
\end{abstract}

Keywords: rectangular pulse, maximum likelihood method, unknown appearance time and duration, joint estimation, single-channel reception, characteristics of estimates.

\section{Introduction}

The estimation problem of the time parameters of pulse signals observed against noise is actual for many applications of radioand hydrolocations, radio communications, seismology etc. [1,2]. In [3] the measurer of the appearance time and duration of a rectangular video pulse, which is synthesized using a maximum likelihood method, is presented. However, its technical implementation is possible as a multichannel device only and the estimates obtained are not consistent with a finite number of measurer channels. In work [4] the more general problem is considered of reception of the signal with stepwise changes at the $M$ random points in time. But, in order to find the estimates of these points even in the simplest case when $M=2$, it is necessary to solve rather complex nonlinear stochastic equations. In [5] the two-channel estimation algorithms of the moments of appearance and disappearance of a rectangular pulse are synthesized based on the maximum likelihood and Bayesian approaches. However, the domain of their applicability is limited to the tasks of localization of an information signal (when the prior interval of possible values of the moment of pulse appearance and disappearance does not exceed two minimum possible pulse durations).

Below we show that using the modification of a maximum likelihood method the very 
simple and easily feasible estimation algorithm of the appearance time and duration of a rectangular video pulse cab be obtained, without restrictions to the domain of like values of unknown parameters.

\section{The synthesis of the estimation algorithm}

So, let the additive mix

$$
x(t)=s\left(t, \lambda_{0}, \tau_{0}\right)+n(t)
$$

is passed to the receiver input during time slice $[0, T]$. Here

$$
\begin{aligned}
& s\left(t, \lambda_{0}, \tau_{0}\right)=a I\left(\frac{t-\lambda_{0}}{\tau_{0}}\right), \\
& I(x)= \begin{cases}1, & |x| \leq 1 / 2 \\
0, & |x|>1 / 2\end{cases}
\end{aligned}
$$

is the useful signal, which is a rectangle video pulse with amplitude $a$, appearance time $\lambda_{0}$ and duration $\tau_{0}$, and $n(t)$ is Gaussian white noise with one-sided spectral density $N_{0}$.

On observable realization (1) it is necessary to estimate the parameters $\lambda_{0}, \tau_{0}$ possessing the values from appropriate prior intervals $\left[\Lambda_{1}, \Lambda_{2}\right],\left[\mathrm{T}_{1}, \mathrm{~T}_{2}\right]$. And we presuppose that for $\Lambda_{1}, \Lambda_{2}$ the following condition is satisfied: $0 \leq \Lambda_{1}-\mathrm{T}_{2} / 2<\Lambda_{2}+\mathrm{T}_{2} / 2 \leq T$, that is pulse (2) is always located within observation interval $[0, T]$.

According to [6] we write down the logarithm of the functional of likelihood ration (FLR) as function of current values $\lambda$, $\tau$ of the unknown parameters $\lambda_{0}, \tau_{0}$ in the form of

$$
L(\lambda, \tau)=\frac{2 a}{N_{0}} \int_{\lambda-\tau / 2}^{\lambda+\tau / 2} x(t) d t-\frac{a^{2} \tau}{N_{0}}
$$

When maximizing Eq. (3) on variables $\lambda, \tau$, we obtain the maximum likelihood estimates (MLEs) of the pulse (2) appearance time and duration defined as [3]

$$
\left(\lambda_{m}, \tau_{m}\right)=\underset{\lambda \in\left[\Lambda_{1}, \Lambda_{2}\right], \tau \in\left[\mathrm{T}_{1}, \mathrm{~T}_{2}\right]}{\arg \sup } L(\lambda, \tau) .
$$

As can be seen, the measurer (4) is multichannel device and the number of channels should be infinite for its exact implementation that is not likely to take place in practice.

Let us consider the possibility to obtain the technically simpler and practically realized estimates of parameters $\lambda_{0}, \tau_{0}$. For this purpose we study the behavior of the logarithm of FLR (3) as function of variable $\lambda$ at the fixed value $\tau=\tau^{*}$. Following [6], we present the functional $L\left(\lambda, \tau^{*}\right)$ as sum of signal $S(\lambda)=\left\langle L\left(\lambda, \tau^{*}\right)\right\rangle \quad$ and noise $N(\lambda)=L\left(\lambda, \tau^{*}\right)-\left\langle L\left(\lambda, \tau^{*}\right)\right\rangle$ functions:

$$
L\left(\lambda, \tau^{*}\right)=S(\lambda)+N(\lambda)
$$

Here \langle\rangle designate the averaging operation on all realizations of the observable data (1) at the fixed values $\lambda_{0}, \tau_{0}$.

From Eq. (3) for the signal function we get

$$
S(\lambda)=2 a^{2} C\left(\lambda-\lambda_{0}\right) / N_{0}-a^{2} \tau^{*} / N_{0},
$$

where

$$
C(\lambda)= \begin{cases}\tau_{0}-\max \left(0, \tau_{0}-\tau^{*}\right), & |\lambda| \leq\left|\tau_{0}-\tau^{*}\right| / 2, \\ \left(\tau_{0}-\tau^{*}\right) / 2-|\lambda|, & \left|\tau_{0}-\tau^{*}\right| / 2<|\lambda| \leq\left(\tau_{0}+\tau^{*}\right) / 2, \\ 0, & |\lambda|>\left(\tau_{0}+\tau^{*}\right) / 2 .\end{cases}
$$


Noise function $N(\lambda)$ is centered Gaussian

random process with correlation function of the kind of

$$
\left\langle N\left(\lambda_{1}\right) N\left(\lambda_{2}\right)\right\rangle=\left(2 a^{2} / N_{0}\right) \max \left[0, \tau^{*}-\left|\lambda_{2}-\lambda_{1}\right|\right] .
$$

As is widely known [6], the logarithm of FLR (3) as well as any biunique instantaneous its transformation is the sufficient statistics with all the information on unknown parameters $\lambda_{0}, \tau_{0}$ which can be extracted from the realization of the observable data (1). As such transformation we apply the derivation (on a variable $\lambda$ ) to Eq. (5). Then we have

$$
M(\lambda)=L^{\prime}\left(\lambda, \tau^{*}\right)=\frac{2 a^{2}}{N_{0}}\left[I\left(\frac{\lambda-\theta_{01}}{\tau_{\min }}\right)-I\left(\frac{\lambda-\theta_{02}}{\tau_{\min }}\right)\right]+\xi(\lambda) .
$$

(6)

Here $\theta_{01}=\lambda_{0}-\tau_{\max } / 2, \theta_{02}=\lambda_{0}+\tau_{\max } / 2$, $\tau_{\max }=\max \left(\tau_{0}, \tau^{*}\right), \tau_{\min }=\min \left(\tau_{0}, \tau^{*}\right), \xi(\lambda)$ is Gaussian white noise with one-sided spectral density $N_{\xi}=8 a^{2} / N_{0}$.

From Eq. (6) follows that when

$$
\tau^{*} \leq \mathrm{T}_{1}
$$

the estimation problem of the appearance time and duration of the pulse (2) can be reduced to the estimation problem of the appearance times of two heteropolar rectangular video pulses by duration $\tau^{*}$ everyone. Indeed, by forming the estimates $\theta_{1 q}, \theta_{2 q}$ of the parameters $\theta_{01}, \theta_{02}$ based on Eqs. (6), for the estimates $\lambda_{q}, \tau_{q}$ of the parameters $\lambda_{0}, \tau_{0}$ we get

$$
\lambda_{q}=\left(\theta_{2 q}+\theta_{1 q}\right) / 2, \quad \tau_{q}=\theta_{2 q}-\theta_{1 q} .
$$

The estimates $\theta_{1 q}, \theta_{2 q}$ can be found by one of possible optimal ways, for example, by means of the correlation reception [6]. Then we have

$$
\theta_{1 q}=\underset{\theta_{1} \in\left[\Lambda_{1}-\tau^{*} / 2, \Lambda_{2}-\tau^{*} / 2\right]}{\operatorname{argsup}} M_{\mathrm{I}}\left(\theta_{1}\right), \theta_{2 q}=\underset{\theta_{2} \in\left[\Lambda_{1}+\tau^{*} / 2, \Lambda_{2}+\tau^{*} / 2\right]}{\operatorname{arginf}} M_{\mathrm{I}}\left(\theta_{2}\right), M_{\mathrm{I}}(\theta)=\int_{\theta-\tau^{*} / 2}^{\theta+\tau^{*} / 2} M(\lambda) d \lambda \text {. (9) }
$$

At the same time, obviously, the following condition should be satisfied

$$
\begin{aligned}
& \tau^{*} \leq \theta_{2 q}-\theta_{1 q} \leq \mathrm{T}_{2} . \\
& \theta_{1 q}=\underset{\theta_{1} \in\left[\Lambda_{1}-\tau^{*} / 2, \Lambda_{2}-\tau^{*} / 2\right]}{\arg \sup } M_{\mathrm{I}}\left(\theta_{1}\right), \quad \theta_{2 q}=\underset{\theta_{2} \in\left[\theta_{1 q}+\mathrm{T}_{1}, \min \left(\theta_{1 q}+\mathrm{T}_{2}, \Lambda_{2}+\mathrm{T}_{1}-\tau^{*} / 2\right)\right]}{\arg \inf } M_{\mathrm{I}}\left(\theta_{2}\right)
\end{aligned}
$$

In order to ensure the fulfillment of inequalities (10) for any sizes of prior domains $\left[\Lambda_{1}, \Lambda_{2}\right],\left[\mathrm{T}_{1}, \mathrm{~T}_{2}\right]$ and signal-to-noise ratios (SNRs), instead of algorithm (9) one of its possible modification can be used, for example,

or

$$
\theta_{1 q}=\underset{\theta_{1} \in\left[\max \left(\Lambda_{1}-\mathrm{T}_{1}+\tau^{*} / 2, \theta_{2 q}-\mathrm{T}_{2}\right), \theta_{2 q}-\tau^{*}\right]}{\arg \sup } M_{\mathrm{I}}\left(\theta_{1}\right), \quad \theta_{2 q}=\underset{\theta_{2} \in\left[\Lambda_{1}+\tau^{*} / 2, \Lambda_{2}+\tau^{*} / 2\right]}{\operatorname{arginf}} M_{\mathrm{I}}\left(\theta_{2}\right) .
$$

The expressions (3), (6), (9), (11) determine the organization of the quasi-optimal measurer of the appearance time and duration of a signal
(2). The block diagram of one of possible measurer implementations is shown in Fig. 1 where are the designations: 1 is the switch that 
is open for time $[0, T], 2$ is an integrator, 3 is a delay line for the period $\tau^{*}, 4$ is the substracter, 5 is the differentiator, 6 and 10 is the retrievers of the location of the input signal greatest maximum and minimum (extremators), respectively, 7 is the summator, 8 is the half-gain amplifier (halver), 9 is the switch that is open for time $\left[\theta_{1 q}+\mathrm{T}_{1}, \min \left(\theta_{1 q}+\mathrm{T}_{2}, \Lambda_{2}+\mathrm{T}_{1}-\tau^{*} / 2\right)\right]$.

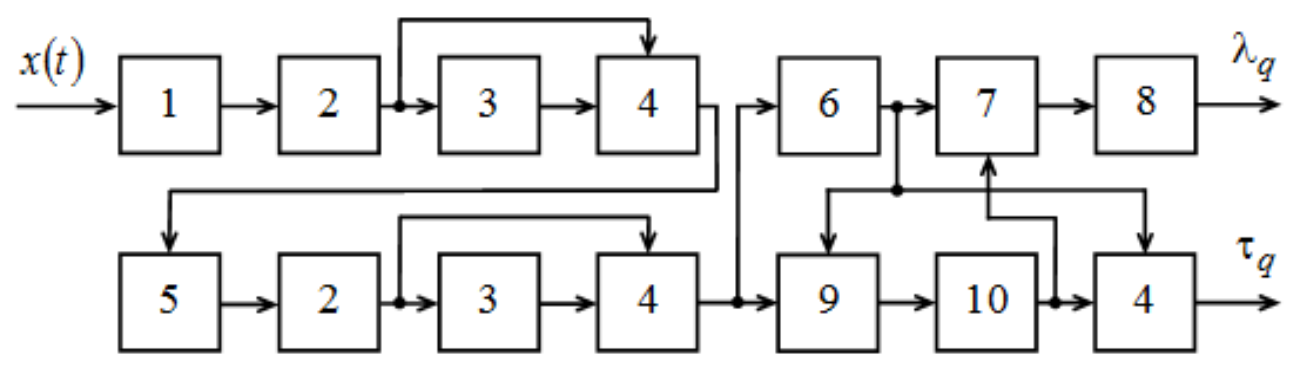

Figure 1. The quasi-optimal measurer of the appearance time and duration of a rectangular pulse.

As shown in Fig. 1, the introduced measurer is only two-channel and technically simpler than known analogues. And the closer $\tau^{*}$ to $\tau_{0}$, this measurer tends to optimal.

\section{The characteristics of the passed estimates}

Let us find the characteristics of the estimates (8). We presuppose that SNR $z^{2}$ for algorithm (11) is great enough, i.e. the following condition is satisfied

$$
z^{2}=S_{I}^{2}\left(\theta_{01}\right) /\left\langle N_{I}^{2}\left(\theta_{01}\right)\right\rangle=S_{I}^{2}\left(\theta_{02}\right) /\left\langle N_{I}^{2}\left(\theta_{02}\right)\right\rangle=a^{2} \tau^{*} / N_{0}=z_{0}^{2} \Delta_{\tau} / 2>>1
$$

Here

$$
S_{I}(\theta)=\left\langle M_{I}(\theta)\right\rangle=\left(2 a^{2} / N_{0}\right)\left[\max \left(0, \tau^{*}-\left|\theta-\theta_{01}\right|\right)-\max \left(0, \tau^{*}-\left|\theta-\theta_{02}\right|\right)\right]
$$

is the signal component, $\left\langle N_{I}^{2}(\theta)\right\rangle=4 a^{2} \tau^{*} / N_{0}$ is the dispersion of the noise component $N_{I}(\theta)=M_{I}(\theta)-\left\langle M_{I}(\theta)\right\rangle$ of the decision statistics (9), $z_{0}^{2}=2 a^{2} \tau_{0} / N_{0}$ is SNR for the optimal receiver of a signal (2) with prior known duration, $\Delta_{\tau}=\tau^{*} / \tau_{0}$ is the relative pulse duration deviation.

According to [7] with increased $z^{2}$, the estimates $\theta_{1 q}, \theta_{2 q}$ (11) converge to true values $\theta_{01}, \theta_{02}$ in mean square. So, if the condition (12) is satisfied, then the estimates $\theta_{1 q}, \theta_{2 q}$ are located in small neighborhoods of

$$
b_{0}\left(\lambda_{q} \mid \lambda_{0}, \tau_{0}\right)=\left[b_{0}\left(\theta_{1 q} \mid \theta_{01}\right)+b_{0}\left(\theta_{2 q} \mid \theta_{02}\right)\right] / 2, \quad b_{0}\left(\tau_{q} \mid \lambda_{0}, \tau_{0}\right)=b_{0}\left(\theta_{2 q} \mid \theta_{01}\right)-b_{0}\left(\theta_{1 q} \mid \theta_{02}\right),
$$




$$
V_{0}\left(\lambda_{q} \mid \lambda_{0}, \tau_{0}\right)=\left[V_{0}\left(\theta_{1 q} \mid \theta_{01}\right)+V_{0}\left(\theta_{2 q} \mid \theta_{02}\right)\right] / 4, \quad V_{0}\left(\tau_{q} \mid \lambda_{0}, \tau_{0}\right)=V_{0}\left(\theta_{2 q} \mid \theta_{02}\right)-V_{0}\left(\theta_{1 q} \mid \theta_{01}\right) .
$$

Using these results for conditional biases and

The characteristics of the estimate of the appearance time of a rectangular video pulse variances of the estimates $\theta_{1 q}, \theta_{2 q}$ we have were studied in a number of works [7-9, etc.].

$$
b_{0}\left(\theta_{1 q} \mid \theta_{01}\right)=b_{0}\left(\theta_{2 q} \mid \theta_{02}\right)=0, \quad V_{0}\left(\theta_{1 q} \mid \theta_{01}\right)=V_{0}\left(\theta_{2 q} \mid \theta_{02}\right)=13 \tau_{0}^{2} / 2 z^{4}=26 \tau_{0}^{2} / z_{0}^{4} \Delta_{\tau}^{2} .
$$

From Eqs. (13), (14) follows that at high conditionally unbiased and possess the conditional variances values of SNR the estimates $\lambda_{q}, \tau_{q}(8)$ are

$$
V_{0}\left(\lambda_{q} \mid \lambda_{0}, \tau_{0}\right)=13 \tau_{0}^{2} / z_{0}^{4} \Delta_{\tau}^{2}, \quad V_{0}\left(\tau_{q} \mid \lambda_{0}, \tau_{0}\right)=52 \tau_{0}^{2} / z_{0}^{4} \Delta_{\tau}^{2}
$$

With fixed values of the signal (2) amplitude and spectral density of white noise the variances (15) is the less when the relative detuning $\Delta_{\tau}$ is close to 1 . Thus, the value $\tau^{*}$ is expedient for choosing as maximum possible, i.e. taking into account Eq. (7) as $\tau^{*}=\mathrm{T}_{1}$. If we take $\Delta_{\tau}=1\left(\tau^{*}=\tau_{0}\right)$ in Eq. (15), then we obtain the expressions for conditional variances of MLEs (4) of the appearance time and duration of a rectangular video pulse without considering the hardware implementation of a measurer (when the number of channels is infinitely large) [3]. We shall note, that the variances of the combined estimates (15) are twice the variances of corresponding separate estimates $[8,10]$, i.e. there is a nonlinear statistical dependence between estimates $\lambda_{q}$ and $\tau_{q}$ [3].

For unconditional variances of the estimates (8) we have

$$
\begin{aligned}
& V_{0}\left(\lambda_{q}\right)=\int_{\Lambda_{1}}^{\Lambda_{2}} \int_{\mathrm{T}_{1}}^{\mathrm{T}_{2}} V_{0}\left(\lambda_{q} \mid \lambda_{0}, \tau_{0}\right) w_{p r}\left(\lambda_{0}, \tau_{0}\right) d \lambda_{0} d \tau_{0}=\int_{\mathrm{T}_{1}}^{\mathrm{T}_{2}} V_{0}\left(\lambda_{q} \mid \lambda_{0}, \tau_{0}\right) w_{p r}\left(\tau_{0}\right) d \tau_{0}, \\
& V_{0}\left(\tau_{q}\right)=\int_{\Lambda_{1}}^{\Lambda_{2}} \int_{\mathrm{T}_{1}}^{\mathrm{T}_{2}} V_{0}\left(\tau_{q} \mid \lambda_{0}, \tau_{0}\right) w_{p r}\left(\lambda_{0}, \tau_{0}\right) d \lambda_{0} d \tau_{0}=\int_{\mathrm{T}_{1}}^{\mathrm{T}_{2}} V_{0}\left(\tau_{q} \mid \lambda_{0}, \tau_{0}\right) w_{p r}\left(\tau_{0}\right) d \tau_{0} .
\end{aligned}
$$

Here it is designated: $w_{p r}\left(\lambda_{0}, \tau_{0}\right)-$ joint probability density of the parameters $\lambda_{0}, \tau_{0}$, $w_{p r}\left(\tau_{0}\right)$ - prior probability density of the parameter $\tau_{0}$ and it is also taken into account that the variances (15) do not depend on $\lambda_{0}$.

If the number of channels $N$ is finite, then similarly to [11] the quality of the estimate $\lambda_{m N}$ of the appearance time of a signal (2) can be characterized its limit (maximum) conditional $V_{0}\left(\lambda_{m N} \mid \lambda_{0}\right)$ or unconditional $V_{0}\left(\lambda_{m N}\right)$ variance, as well as the quality of the estimate $\tau_{m N}$ of the duration - the conditional $V_{0}\left(\tau_{m N} \mid \tau_{0}\right)$ or unconditional $V_{0}\left(\tau_{m N}\right)$ variance of the estimate for multichannel reception. We introduce the following designations: $\tau_{i}=\mathrm{T}_{1}+(i-1 / 2) \Delta, i=\overline{1, N}$ is the duration, to which $i$-th channel is tuned, $\Delta=\left(\mathrm{T}_{2}-\mathrm{T}_{1}\right) / N$ is the distance between the two adjacent channels, $\delta=\left(\mathrm{T}_{2}-\mathrm{T}_{1}\right) /\left(\mathrm{T}_{2}+\mathrm{T}_{1}\right)$ is the maximum possible absolute deviation of the signal duration $\tau_{0}$ (2) from the average duration $\tau_{c}=\left(\mathrm{T}_{2}+\mathrm{T}_{1}\right) / 2, z_{c}^{2}=2 a^{2} \tau_{c} / N_{0}$ is SNR for a 
pulse (2), the duration of which is equal to average duration $\tau_{c}, w\left(\tau_{m} \mid \tau_{0}\right)$ is the conditional probability density of MLE $\tau_{m}$ (4) in the measurer with the continuous change of current value of the estimated parameter. Then, in terms of the results $[11,12]$, we have

$$
\begin{aligned}
& V_{0}\left(\lambda_{m N} \mid \lambda_{0}\right)=V_{0}\left(\lambda_{m N}\right)=\frac{\delta^{2}}{8 N^{2}}+\exp \left(\frac{z_{c}^{2} \delta}{N}\right)\left[1-\Phi\left(z_{c} \sqrt{\frac{2 \delta}{N}}\right)\right] \times \\
& \times\left(\frac{13}{z_{c}^{4}}-\frac{13 \delta}{z_{c}^{2} N}+\frac{8 \delta^{2}}{N}-\frac{4 z_{c}^{2} \delta^{3}}{3 N^{3}}\right)+\sqrt{\frac{\delta}{\pi N}\left(\frac{2 z_{c} \delta^{2}}{3 N^{2}}-\frac{13 \delta}{3 z N}+\frac{13}{z_{c}^{3}}\right),} \\
& V_{N}\left(\tau_{m N} \mid \tau_{0}\right)=\sum_{i=-(v-1)}^{N-v}(i \Delta)^{2} \int_{\mathrm{T}_{1}+(v+i) \Delta}^{\mathrm{T}_{1}+(v+i-1) \Delta} w\left(\tau_{m} \mid \tau_{0}\right) d \tau_{m}, \\
& V_{N}\left(\tau_{m N}\right)=\sum_{i=-(N-1)}^{N-1}(i \Delta)^{2} \sum_{v=1}^{N} \int_{\mathrm{T}_{1}+(v-1) \Delta}^{\mathrm{T}_{1}+v \Delta} w_{p r}\left(\tau_{0}\right) \int_{\mathrm{T}_{1}+(v+i-1) \Delta}^{\mathrm{T}_{1}+(\mathrm{v}+i) \Delta} w\left(\tau_{m} \mid \tau_{0}\right) d \tau_{m} d \tau_{0} .
\end{aligned}
$$

In Eq. (18) the number of the channel, nearest to $\tau_{0}$, is meant by $v$.

The probability density $w\left(\tau_{m} \mid \tau_{0}\right)$ can be written down through the joint probability density $w\left(\lambda_{m}, \tau_{m} \mid \lambda_{0}, \tau_{0}\right)$ of the estimates (4) found in [3] as

$$
w_{2}\left(\lambda_{m}, \tau_{m} \mid \lambda_{0}, \tau_{0}\right)=\frac{w_{1}\left[\lambda_{m}-\lambda_{0}-\left(\tau_{m}-\tau_{0}\right) / 2\right] w_{1}\left[\lambda_{m}-\lambda_{0}+\left(\tau_{m}-\tau_{0}\right) / 2\right]}{\int_{\Lambda_{1}}^{\Lambda_{2} T_{1}} w_{1}\left[\lambda_{m}-\lambda_{0}-\left(\tau_{m}-\tau_{0}\right) / 2\right] w_{1}\left[\lambda_{m}-\lambda_{0}+\left(\tau_{m}-\tau_{0}\right) / 2\right] d \lambda_{m} d \tau_{m}},
$$

where

$$
\begin{aligned}
& w_{2}\left(\lambda_{m}, \tau_{m} \mid \lambda_{0}, \tau_{0}\right)=\frac{w_{1}\left[\lambda_{m}-\lambda_{0}-\left(\tau_{m}-\tau_{0}\right) / 2\right] w_{1}\left[\lambda_{m}-\lambda_{0}+\left(\tau_{m}-\tau_{0}\right) / 2\right]}{\int_{\Lambda_{1}}^{\Lambda_{2} T_{1}} \int_{1} w_{1}\left[\lambda_{m}-\lambda_{0}-\left(\tau_{m}-\tau_{0}\right) / 2\right] w_{1}\left[\lambda_{m}-\lambda_{0}+\left(\tau_{m}-\tau_{0}\right) / 2\right] d \lambda_{m} d \tau_{m}}, \\
& w_{1}(x)=\left(z_{0}^{2} / 2 \tau_{0}\right) w\left(z_{0}^{2} x / 2 \tau_{0}\right), \quad w(x)=\Phi(\sqrt{|x| / 2})-1+3 \exp (2|x|)[1-\Phi(3 \sqrt{|x| / 2})] .
\end{aligned}
$$

It should be noted that unlike the quasi-optimal estimates (8), the MLEs of the appearance time and duration (4) of a signal (2) are not consistent with a finite number of channels $N$.

The expressions (15)-(19) allow us to assess the reasonableness of the application of this or that estimation algorithm of time parameters of a rectangular video pulse depending on the available prior information and the required accuracy and simplicity of hardware measurer implementation.

\section{Conclusion}

In order to jointly estimate the several parameters of information signals the technique based on additional transformation of the logarithm of the functional of likelihood ratio can be used in some cases. The purpose of the specified transformation is to divide a regular component of decision statistics into the components depending on one parameter, 
to carry out a separate estimation of parameters and to find the values of required parameters by the found separate estimates. Quasi-optimal measurers obtained in that way are technically simpler in comparison with optimal (maximum likelihood) analogues and can provide higher performance in actual conditions. The synthesis of two-channel estimation algorithm of the appearance time and duration of a rectangular video pulse represented in the work confirms the efficiency and usefulness of the introduced approach in practical applications.

\section{Acknowledgement}

This research was financially supported by the Russian Science Foundation (research project No. 14-49-00079).

\section{References}

[1] A.G. Tartakovskii, Detection of signals with random moments of appearance and disappearance, Problems of Information Transmission, 2 (1988) 115-124.

[2] V.G. Repin, Detection of a signal with unknown moments of appearance and disappearance, Problems of Information Transmission, 1 (1991) 49-58.

[3] A.P. Trifonov, V.K. Buteyko, Joint estimation of two parameters of an analog signal against a white-noise background, Soviet Journal of Communications Technology \& Electronics, 12 (1990) 25-32.

[4] A.A. Mal'tsev, A.M. Silaev, Optimum estimates of the times of random irregular variations in signal parameters, Soviet journal of communications technology \& electronics, 13 (1989) 116-124.

[5] A.P. Trifonov, Yu.E. Korchagin, Optimal reception of a rectangular pulse with unknown times of appearance and disappearance, Izvestiya Vysshikh Uchebnykh Zavedenij. Radiofizika, 3 (2000) 271-282.

[6] E.I. Kulikov, A.P. Trifonov, Estimation of Signal Parameters Against Hindrances (in Russian), Sovetskoe Radio, Moscow, 1978.
[7] A.P. Trifonov, Yu.S. Shinakov, Joint Discrimination of Signals and Estimation of Their Parameters against Background (in Russian), Radio I Svyaz', Moscow, 1986.

[8] A.S. Terent'ev, Probability distribution of the absolute maximum time position at the output of matched filter. Radiotekhnika I Elektronika, 4 (1968), 652-657.

[9] O.V. Chernoyarov, Sai Si Thu Min, A.V. Salnikova, B.I. Shakhtarin, A.A. Artemenko, Application of the local Markov approximation method for the analysis of information processes processing algorithms with unknown discontinuous parameters, Applied Mathematical Sciences, 90 (2014) 4469-4496.

[10]A.P. Trifonov, Yu.E. Korchagin, Receiving a signal with unknown duration, Izvestiya Vysshikh Uchebnykh Zavedenij. Radiofizika, 7 (2002) 625-637.

[11]O.V. Chernoyarov, M. Vaculik, A.E. Rozanov, A new calculation technique of characteristics of multichannel measurer, Science Journal of Circuits, Systems and Signal Processing, 3 (2013) 78-84.

[12] O.V. Chernoyarov, Sai Si Thu Min, Yu.A. Guseva, B.I. Shakhtarin, A.A. Artemenko, Application of the local Markov approximation method for the analysis of information processes processing algorithms with unknown discontinuous parameters under violation of the consistency property of their estimates, Applied Mathematical Sciences, 126 (2014) 6267-6294. 